\title{
Acute Schmorl Node in Dorsal Spine: An Unusual Cause of a Sudden Onset of Severe Back Pain in a Young Female
}

\author{
Sara Abu-Ghanem ${ }^{1}$, Nissim Ohana ${ }^{2}$, Yasmin Abu-Ghanem ${ }^{1}$, Mohamed Kittani ${ }^{2}$, Ilan Shelef ${ }^{1}$ \\ ${ }^{1}$ Department of Diagnostic Imaging, Soroka University Medical Center, Faculty of Health Sciences Ben Gurion University, Beer Sheva, Israel \\ ${ }^{2}$ Department of Orthopedic Surgery, Rabin Medical Center (Beilinson Campus), Petah Tikva, Israel
}

\begin{abstract}
Schmorl nodes represent displacement of intervertebral disc tissue into the vertebral body and have been considered as an asymptomatic incidental radiological finding on plain radiographs, computed tomography and magnetic resonance imaging (MRI). Although uncommon, acute symptomatic Schmorl nodes causing severe back pain do occur. We report here an unusual case of acute painful Schmorl node in a young healthy woman, with no previous trauma, presenting with a sudden significant localized back pain within hours accompanied by characteristic findings on a MRI scan. We reviewed all reports of symptomatic Schmorl nodes known in the literature, focusing mainly on MRI findings, and recent treatment options.
\end{abstract}

Keywords: Magnetic resonance imaging; Acute painful Schmorl node; Intravertebral disc herniation

\section{Introduction}

Schmorl nodes, classically known as intravertebral disc herniations, were first described by Schmorl in 1927 [1]. Schmorl nodes represent a herniation or an extrusion of the intervertebral disc nuclear material through the vertebral body endplate, with displacement of this material into the adjacent vertebral body [2]. The herniated tissue may form a defect in the upper or lower surface of the involved vertebra. The lesions tend to occur at the central or posterior axis of the vertebrae, near the thoracolumbar junction $[3,4]$.

Trauma or stress transmitted through a weakened endplate is the proposed pathogenesis of Schmorl's node formation [4]. This could be due to intrinsic factors in the endplate (indentations, ossification gaps, vascular channels, Scheuermann's disease) or acquired factors (infection, malignancy, osteoporosis or osteomalacia, hyperparathyroidism, Paget's disease) [4,5]. However, such weakening of the endplate is not a necessary precondition for extrusion and is thought to be present as an underlying cause only in a small percentage of Schmorl node cases. Most Schmorl nodes form after axial-loading trauma results in the preferential extrusion of nuclear material through the vertebral endplate, rather than through an intact, normal annulus fibrosus [6].

Schmorl nodes are generally considered to be an asymptomatic incidental finding. To date, there were some reports of symptomatic Schmorl nodes, however, only a minority of them presented cases of acute onset of back and neck pain associated with the lesion [5,7-21].

In the current report, we present an unusual case of

Received Apr 15, 2012; Revised May 15, 2012; Accepted May 16, 2012

Corresponding author: Sara Abu-Ghanem

Department of Diagnostic Imaging, Soroka University Medical Center, PO Box 151 Beer Sheva 84105, Israel

Tel: +972-8-6400236, Fax: +972-8-6364087, E-mail: abughane@gmail.com 
an acute painful Schmorl node in a young woman with no previous history of trauma, presenting with a sudden significant localized back pain within hours, and accompanied by characteristic findings on a magnetic resonance imaging (MRI) scan. We reviewed all the reports of symptomatic Schmorl nodes known in the literature, focusing mainly on MRI findings, and recent treatment options.

\section{Case Report}

A 23-year-old, otherwise healthy Caucasian woman was admitted to a spine outpatient clinic with severe symptoms of acute localized back pain, which had appeared suddenly the previous night. The patient had no previous history of back pain, nor history of significant compression/flexion stress to the spine, including any kind of trauma. She denied paraesthesias, numbness, or weakness. All clinical findings were normal apart from restriction of back motion due to intense back pain. No neurological abnormalities were noted, and routine laboratory results were found to be normal. Furthermore, plain film of the lumbar spine showed no abnormalities.

Due to its sudden appearance and the severity of the symptoms, further imaging studies were performed. Sagittal T2-weighted MRI showed an impressive Schmorl node in the T11 vertebral body (Fig. 1A, arrow) surrounded by a marked bone marrow oedema of the whole vertetebra body. Diffused, homogeneous, low signal intensity on sagittal T1-weighted images (Fig. 1B, arrow), diffused enhancement of both Schmorl node and vertebra is noted on T1-weighted with fat saturation (Fig. 1C). The patient was treated conservatively by bed rest and analgesic medications. Two weeks later the patient was symptom-free. A routine follow-up revealed no residual abnormalities, and the patient continues to enjoy good health.

\section{Discussion}

Nonacute asymptomatic Schmorl nodes are common spinal abnormalities and are reported to occur in 38\% to $75 \%$ of the population, with male predominance $[22,23]$. The variation in prevalence could be attributed to several factors: differences in assessment methodologies (i.e., method of investigation; number of vertebrae examined; which vertebrae and which vertebral surface was observed, superior, inferior or both), subject inclu-
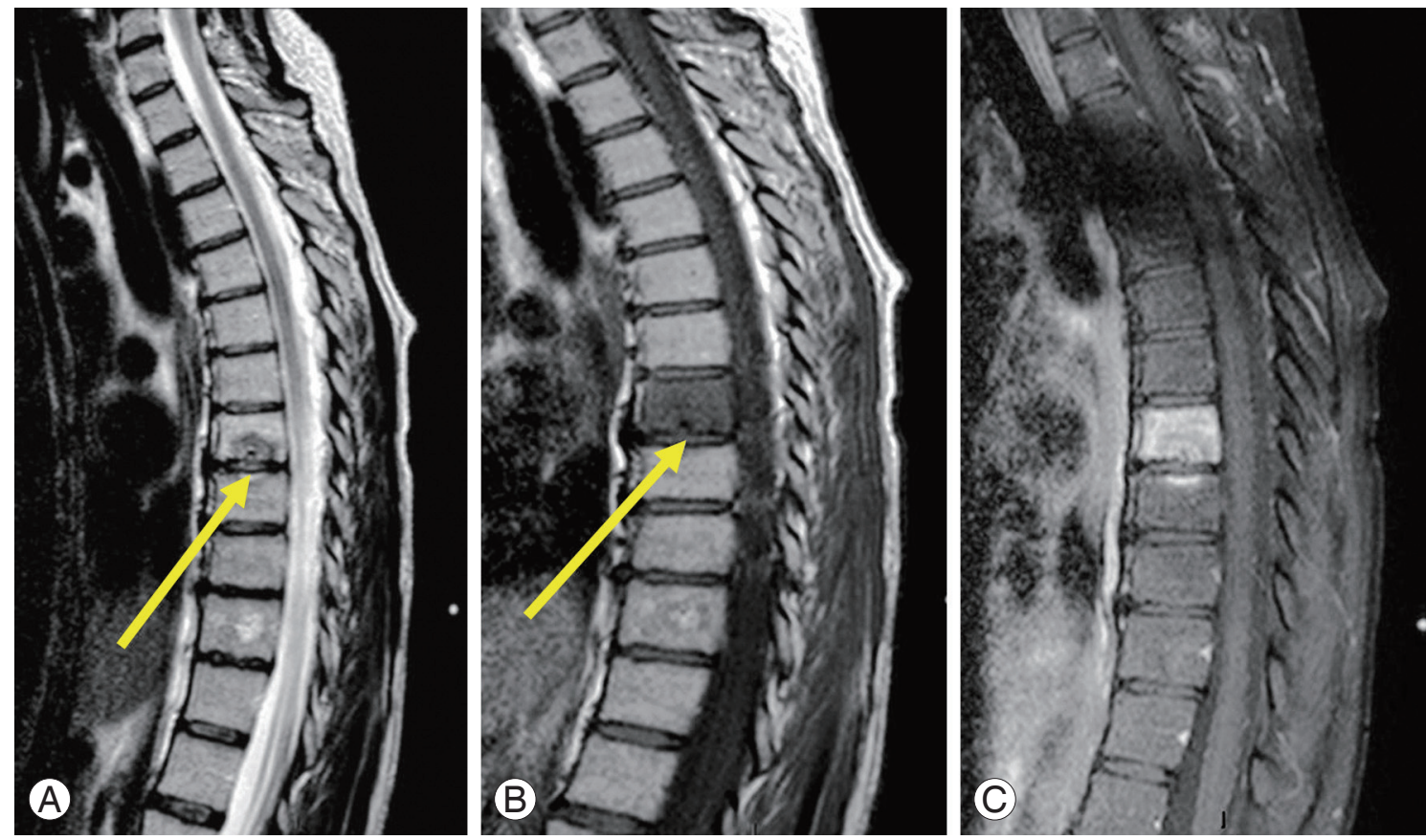

Fig. 1. (A) Sagittal T2-weighted images demonstrate a large schmorl node (arrow) surrounded by bone marrow edema (high signal intensity). (B) Sagittal T1-weighted images demonstrate low signal intensity (arrow) due to diffused bone marrow edema. (C) Sagittal T1-weighted images with fat saturation demonstrate enhancment of both the node and the edematous bone marrow, minimal enhancement is noted at the adjacent lower vertebra. 
sion criteria (i.e., definition of "individuals with Schmorl nodes": one or multiple cases of Schmorl nodes), including demographics (sex ratio, ethnic origin, etc.), and socioeconomic characteristics (mainly daily activities) of the examined population $[1,24]$.

The Schmorl node may be well demonstrated at plain radiography, computed tomography $(\mathrm{CT})$, and was recently demonstrated by bone scintigraphy [21]. However, MRI is the modality of choice for the diagnosis of symptomatic Schmorl nodes as plain film radiographs and CT do not differentiate symptomatic from asymptomatic nodes, and the radionuclide bone scan is not specific.

The detection of Schmorl nodes on conventional radiographs depends on the size of the nodes as well as the reactive processes, such as fibrosis and sclerosis, in the adjacent trabecular bone. Coventry et al. first reported in 1945 that only $3.6 \%$ of 55 pathologically confirmed Schmorl's nodes were visible on conventional X-ray and in a 1988 study, Yasuma et al. reported that $5.6 \%$ of 54 nodes identified histologically were visible with conventional radiography [25]. Hamanishi et al. [26] found that X-ray revealed only $33 \%$ of nodes visualized with MRI. Therefore, plain radiographs have limited value in assessing Schmorl nodes, and especially acute Schmorl nodes. In contrast, vascularization and bone marrow reaction with increased free water can be seen only with MRI [15]. Furthermore, it has been shown that the signal changes on MRI are reflective of bone marrow edema and inflammation seen on histology.

Most consider Schmorl nodes to be asymptomatic, since they are a frequent finding in persons without back pain [27]. However, Hamanishi et al. [26] compared the findings of MRI examinations of the lumbar spine in 400 patients with low back pain with those of a control group of 106 patients and found a significantly higher frequency of Schmorl nodes in the symptomatic group (19\%) in comparison with the control group (9\%).

Takahashi et al. [14], Walters et al. [12], and Stabler et al. [15], showed that in symptomatic patients, the vertebral body marrow surrounding the Schmorl node gave a low signal intensity on T1-weighted sequences and a high signal intensity on T2-weighted and short tau inversion recovery sequences. Therefore, there may be dramatic adjacent vertebral changes of fatty marrow replacement or sclerosis, even extending throughout the vertebral body and into the pedicles, and may involve vertebral bodies on either side of the affected disc $[14,16]$. These MRI findings were not present or present to a lesser extent in the asymptomatic group, which suggested that Schmorl nodes became asymptomatic when the inflammation subsided. Moreover, it was found that these features usually decrease in 3 to 12 months [7]. In the current report, Schmorl nodes were not visible on plain radiography. However, MRI findings of our patient corresponded with acute Schmorl nodes.

Although the presence and pattern of contrast enhancement may be helpful, when the Schmorl node is recent, it can be difficult to differentiate benign degenerative bone disease from malignant infiltration or infection. Moreover, neoplastic and infectious processes may weaken the structural integrity of the supporting trabecular bone, making Schmorl node formation more likely. If the radiologist is aware of the morphologic characteristics of the endplate defect and adjacent disk, MRI is usually sufficient for reliable differentiation and helpful in equivocal cases. The key to Schmorl node diagnosis is the recognition of the herniated disc material and the MRI key features that were described above [12,14-16]. In cases in which marrow oedema is demonstrated extending from the endplate in a vertebral body or two adjacent vertebral bodies without collapse or paraspinal mass, the possibility of acute intraosseous disc herniation must be considered [16].

Controversy also exists regarding the location of Schmorl nodes. Recently, Mok et al. [1] reported that in a cross-sectional population-based MRI study of 2,449 individuals the majority of Schmorl nodes are located in the upper lumbar levels with the highest prevalence in L2/3. While, Dar et al. [24] showed in a skeletal study that Schmorl nodes appear more frequently in the T7L1 region. This finding was consistent with a previous report by Pfirrmann and Resnick [28]. This distribution of Schmorl nodes cannot be explained solely by the differences in the load magnitude along the spine. If that was the case we would expect an increasing prevalence of Schmorl nodes from T1 to L5 (maximum load). Therefore, the higher prevalence of Schmorl nodes in the thoracolumbar region suggests that other factors might be involved. Dar et al. [24] also showed, in concordance with several previous reports, that Schmorl nodes are more common on the inferior surface of the thoracic vertebrae (T4-T11) and on the superior surface of the lumbar vertebrae (L1-L5). To date, there is no convincing explanation for this phenomenon. 
An acute painful Schmorl node is usually treated by conservative therapy with analgesic drugs, bed rest, and bracing; in those cases in which medical therapy is ineffective, and the patient still suffers from persistent disabling back pain, some authors propose surgical treatment. Hasegawa et al. [19] reported a case of a patient with painful Schmorl node treated with eradication of the intervertebral disc including the Schmorl's node and segmental fusion. Masala et al. [20] suggested a vertebroplasty procedure for painful Schmorl nodes that are refractory to medical or physical therapy. And, Jang et al. [5] reported recently a reduction of pain by blocking the ramus communicans nerve in a patient with symptomatic Schmorl node.

In conclusion, the case presented here represents an unusual and painful intravertebral disc herniation in a young woman. Awareness that an acute Schmorl node may be a cause for acute back pain could facilitate an accurate early diagnosis, even though the therapeutic regimen may not change as long as no biomechanical instability is implied. The diagnosis may occasionally be possible from plain radiographs or CT scans, however, the diagnostic tool of choice is MRI. Lastly, acute Schmorl nodes should not be confused with tumor or infection.

\section{Conflict of Interest}

No potential conflict of interest relevant to this article was reported.

\section{References}

1. Mok FP, Samartzis D, Karppinen J, Luk KD, Fong DY, Cheung KM. ISSLS prize winner: prevalence, determinants, and association of Schmorl nodes of the lumbar spine with disc degeneration: a populationbased study of 2449 individuals. Spine (Phila Pa 1976) 2010;35:1944-52.

2. Pilet B, Salgado R, Van Havenbergh T, Parizel PM. Development of acute schmorl nodes after discography. J Comput Assist Tomogr 2009;33:597-600.

3. Williams FM, Manek NJ, Sambrook PN, Spector TD, Macgregor AJ. Schmorl's nodes: common, highly heritable, and related to lumbar disc disease. Arthritis Rheum 2007;57:855-60.

4. Wu HT, Morrison WB, Schweitzer ME. Edematous
Schmorl's nodes on thoracolumbar MR imaging: characteristic patterns and changes over time. Skeletal Radiol 2006;35:212-9.

5. Jang JS, Kwon HK, Lee JJ, Hwang SM, Lim SY. Rami communicans nerve block for the treatment of symptomatic Schmorl's nodes: a case report. Korean J Pain 2010;23:262-5.

6. Fahey V, Opeskin K, Silberstein M, Anderson R, Briggs C. The pathogenesis of Schmorl's nodes in relation to acute trauma: an autopsy study. Spine (Phila Pa 1976) 1998;23:2272-5.

7. Grive E, Rovira A, Capellades J, Rivas A, Pedraza S. Radiologic findings in two cases of acute Schmorl's nodes. AJNR Am J Neuroradiol 1999;20:1717-21.

8. Smith DM. Acute back pain associated with a calcified Schmorl's node: a case report. Clin Orthop Relat Res 1976;(117):193-6.

9. Lipson SJ, Fox DA, Sosman JL. Symptomatic intravertebral disc herniation (Schmorl's node) in the cervical spine. Ann Rheum Dis 1985;44:857-9.

10. McCall IW, Park WM, O’Brien JP, Seal V. Acute traumatic intraosseous disc herniation. Spine (Phila $\mathrm{Pa}$ 1976) $1985 ; 10: 134-7$.

11. Kornberg M. MRI diagnosis of traumatic Schmorl's node: a case report. Spine (Phila Pa 1976) 1988;13:934-5.

12. Walters G, Coumas JM, Akins CM, Ragland RL. Magnetic resonance imaging of acute symptomatic Schmorl's node formation. Pediatr Emerg Care 1991;7:294-6.

13. Takahashi K, Takata K. A large painful Schmorl's node: a case report. J Spinal Disord 1994;7:77-81.

14. Takahashi K, Miyazaki T, Ohnari H, Takino T, Tomita K. Schmorl's nodes and low-back pain. Analysis of magnetic resonance imaging findings in symptomatic and asymptomatic individuals. Eur Spine J 1995;4:569.

15. Stabler A, Bellan M, Weiss M, Gartner C, Brossmann J, Reiser MF. MR imaging of enhancing intraosseous disk herniation (Schmorl's nodes). AJR Am J Roentgenol 1997;168:933-8.

16. Seymour R, Williams LA, Rees JI, Lyons K, Lloyd DC. Magnetic resonance imaging of acute intraosseous disc herniation. Clin Radiol 1998;53:363-8.

17. Wagner AL, Murtagh FR, Arrington JA, Stallworth D. Relationship of Schmorl's nodes to vertebral body endplate fractures and acute endplate disk extrusions. 
AJNR Am J Neuroradiol 2000;21:276-81.

18. Coulier B, Ghosez JP. Lumbar radiculopathy caused by a tunneling transvertebral Schmorl's node. Skeletal Radiol 2002;31:484-7.

19. Hasegawa K, Ogose A, Morita T, Hirata Y. Painful Schmorl's node treated by lumbar interbody fusion. Spinal Cord 2004;42:124-8.

20. Masala S, Pipitone V, Tomassini M, Massari F, Romagnoli A, Simonetti G. Percutaneous vertebroplasty in painful schmorl nodes. Cardiovasc Intervent Radiol 2006;29:97-101.

21. Crawford BA, van der Wall H. Bone scintigraphy in acute intraosseous disc herniation. Clin Nucl Med 2007;32:790-2.

22. Resnick D, Niwayama G. Intravertebral disk herniations: cartilaginous (Schmorl's) nodes. Radiology 1978;126:57-65.

23. Hilton RC, Ball J, Benn RT. Vertebral end-plate lesions (Schmorl's nodes) in the dorsolumbar spine. Ann Rheum Dis 1976;35:127-32.
24. Dar G, Masharawi Y, Peleg S, et al. Schmorl's nodes distribution in the human spine and its possible etiology. Eur Spine J 2010;19:670-5.

25. Silberstein M, Opeskin K, Fahey V. Spinal Schmorl's nodes: sagittal sectional imaging and pathological examination. Australas Radiol 1999;43:27-30.

26. Hamanishi C, Kawabata T, Yosii T, Tanaka S. Schmorl's nodes on magnetic resonance imaging. Their incidence and clinical relevance. Spine (Phila Pa 1976) 1994;19:450-3.

27. Jensen MC, Brant-Zawadzki MN, Obuchowski N, Modic MT, Malkasian D, Ross JS. Magnetic resonance imaging of the lumbar spine in people without back pain. N Engl J Med 1994;331:69-73.

28. Pfirrmann CW, Resnick D. Schmorl nodes of the thoracic and lumbar spine: radiographic-pathologic study of prevalence, characterization, and correlation with degenerative changes of 1,650 spinal levels in 100 cadavers. Radiology 2001;219:368-74. 